\title{
Maternal HIV infection and preterm delivery outcomes at an urban district hospital in KwaZulu-Natal 2011
}

\author{
Mergan Naidoo ${ }^{a *}$, Benn Sartorius ${ }^{b}$ and Guy Tshimanga-Tshikala ${ }^{a}$ \\ aDiscipline of Family Medicine, School of Nursing and Public Health, University of KwaZulu-Natal, Durban, South Africa \\ ${ }^{b}$ Discipline of Public Health Medicine, School of Nursing and Public Health, University of KwaZulu-Natal, Durban, South Africa \\ *Corresponding author, email: naidoom@ukzn.ac.za
}

Background: The seroprevalence of human immunodeficiency virus (HIV) in pregnant women attending public facilities in KwaZulu-Natal province in 2011 was 37\%, the highest in South Africa. An association between the HIV status of the pregnant women and preterm delivery, with associated poorer clinical outcomes, has been reported in many studies. The aim of this study was to explore the relationship between preterm delivery and maternal HIV infections at an urban district hospital in KwaZuluNatal in 2011.

Method: The associations and outcomes of preterm deliveries at a high patient load urban district hospital were determined using a retrospective cohort study.

Results: Of the 250 consecutively enrolled patients, 23 women (9\%) [95\% confidence interval (CI): 5.90-13.50] delivered 25 preterm infants. Ninety-two women (37\%) (95\% Cl: 30.80-43.10) were found to be HIV positive, of whom 13 (14\%) $(95 \% \mathrm{Cl}: 7.70-$ 23.00) delivered preterm neonates, compared to 10 of the 158 women (6\%) (95\% Cl: 3.10-11.30) of the HIV-negative mothers. HIV-positive mothers were significantly more likely $(\sim 4$ fold $)$ to have preterm deliveries than their HIV-negative counterparts, following multivariable adjustment [odds ratio (OR) 4.09, 95\% Cl: 1.37-12.17] ( $p$ 0.010). Hypertension was the only other risk factor to remain significantly associated with preterm delivery following multivariable adjustment (OR $0.07,95 \% \mathrm{Cl}: 0.02-0.28)$ $(p<0.001)$.

Conclusion: The risk of preterm delivery is elevated in HIV-infected pregnant women. HIV-positive pregnant women should be targeted for improved antenatal care and intensive counselling.

Keywords: HIV infection, pre-term delivery

\section{Introduction}

Human immunodeficiency virus (HIV) is a major public health challenge in South Africa, with a prevalence of $10 \%$ in the general population, and $16 \%$ in all people aged $15-49$ years old. ${ }^{1}$ The seroprevalence of HIV in pregnant women attending public facilities in KwaZulu-Natal province in 2011 was 37\%, the highest in South Africa and globally. ${ }^{2}$

\section{HIV and preterm delivery}

The delivery of preterm neonates is a major public health problem in low- and middle-income countries. Causes of preterm delivery are multifactorial, but infection in the form of urinary tract infections, malaria, bacterial vaginosis, syphilis and HIV are significant ones. ${ }^{3}$ The results of a cross-sectional study and a nested case-control study suggested that HIV infection, maternal undernutrition, infrequent antenatal care attendance, not receiving intermittent preventive treatment against malaria and chorioamnionitis are important causes of preterm delivery in this sub-Saharan African population. ${ }^{4}$ Preterm birth rates range from $8 \%$ in high-income countries to $18 \%$ in low- and middle-income countries. ${ }^{1}$ Ellis et al. demonstrated that parturients who were infected with HIV were at increased risk of delivering low-birthweight and premature infants in inner city hospitals in the USA. ${ }^{5} \mathrm{HIV}$-positive pregnant women on prevention of mother-to-child transmission of HIV programmes were found to significantly undergo more preterm deliveries than their HIV-negative counterparts (13\% vs. $6 \%)(p<0.050){ }^{6}$ Intrauterine infection was found to be less of a risk factor for preterm delivery than maternal HIV infection.? Ureaplasm urealyticum co-infection was also found to be high in HIV-infected pregnant women, resulting in an increased predisposition towards preterm deliveries. ${ }^{8}$

\section{Outcomes for preterm infants}

Preterm delivery is also a significant risk factor for neonatal death. ${ }^{3}$ The southern African region was reported to have the highest incidence of preterm deliveries, with an preterm birth rate of $18 \%$ [95\% confidence interval (Cl): 14.60-20.30].' Ryder et al. showed that there was a significantly higher rate of morbidity and mortality in HIV-exposed preterm neonates than in the unexposed control group. ${ }^{9} \mathrm{~A}$ link between maternal HIV infection and preterm delivery has been demonstrated in studies carried out in high-, and low- and middle-income countries, with worse outcomes reported in low- and middle-income countries. ${ }^{10-12}$ Epalza et al. observed that HIV-exposed, but uninfected, infants, were more susceptible to group B streptococcal infection. ${ }^{7}$ Flang et al. found that the mortality risk for premature infants was 2.87 times that of mature infants $(95 \% \mathrm{Cl}: 1.12-7.35)$, while the mortality risk of HIV-infected infants was 9.87 times that of HIVuninfected infants (95\% Cl: 3.81-25.62), indicating that HIVinfected preterm infants experienced a significantly higher mortality risk. ${ }^{13}$

Being a preterm neonate is an additional risk factor for increased morbidity and mortality, and the care of these neonates is costly to the health system. ${ }^{4,14}$

Perinatal data from the urban district hospital in this study reported a very high HIV seroprevalence rate of $38 \%$ in 2010, and while most women carry to full term, the facility is not equipped to provide care for a large number of preterm deliveries. A higher rate of infant mortality in preterm neonates was also seen in the perinatal data from the hospital. Few studies have been 
performed in the local context in which the relationship between maternal HIV status and preterm delivery has been assessed. The labour ward statistics at this local district hospital in South Africa for the period January to December 2008 revealed that an average of 500 deliveries occurred monthly, and that 29 of these deliveries were preterm, i.e. $6 \%$ on average.

The aim of this study was to explore the relationship between HIV infection and prematurity at an urban district hospital in KwaZulu-Natal. The study compared risk factors and outcomes between preterm and term mothers.

\section{Method}

A retrospective observational, cohort design was used. The study population was pregnant women who delivered at an urban district hospital in July 2011. Only women with known HIV status were included in the study. A consecutive sample of 250 patients who met the inclusion criteria was reviewed. Labour ward and patients' medical records were reviewed. A gestational age of $\leq$ 37 completed weeks was used to define preterm delivery. Gestational age was estimated by using the first day of the last menstrual cycle, corroborated with symphysis to fundal height measurements and ultrasound findings. The district hospital included in this study contains a fully functional nursery with dedicated medical and nursing staff, so considerably more preterm infants are managed at this hospital than would be the case at a normal district hospital. Women with a gestational age of 26-32 weeks are normally referred to the regional hospital if a bed is available. In addition, women with maternal disease, such as severe pre-eclampsia, cardiac disease and diabetes mellitus, are referred to the regional hospital. Women with severe maternal disease are referred to the regional hospital when the diagnosis is made at any gestational age. The demographic and clinical data and the outcomes of the preterm neonates were reviewed. Maternal condition was assessed with respect to HIV and preterm status, mode of delivery, HIV status and gestational
The data were analysed using Stata ${ }^{\circledR}$ version $13.0 .{ }^{15}$ Significant differences in preterm delivery risk and perinatal risk by HIV status were assessed using standard Pearson's chi-square $\left(\chi^{2}\right)$ test. If small numbers were evident, i.e. the expected cell with less than five observations, then Fisher's exact test was preferred. Variables significantly associated with the outcome at the 10\% level were selected for multivariable logistic regression. Adjusted ORs and $p$-values are presented. Model fit and diagnostics were performed to ensure statistical validity.

The protocol was approved by the Biomedical Research Ethics Committee of the University of KwaZulu-Natal. Gatekeeper permission was obtained from the Provincial Department of Health, the Head of the Department of Obstetrics and Gynaecology, and the hospital manager of the hospital concerned.

\section{Results}

\section{Mothers' condition}

Ninety-two women (37\%) were HIV positive, of whom 13 (14\%) (95\% Cl: 7.70-23.00) had a pre-term infant, compared to $10(6 \%)$ (95\% Cl: $3.10-11.30$ ) in the HIV-negative group. This difference was statistically significant ( $\chi^{2}$ test, $p=0.040$ ). Of the 23 women who delivered preterm infants, two had twins, yielding a total number of 25 preterm infants. Seventy-three (29\%) of pregnant women delivered by Caesarean section, of whom 22 (9\%) were HIV positive, and 51 (20\%) HIV negative. Of the HIV-positive women who had preterm deliveries, 9 (69\%) were on dual therapy, and 4 (31\%) were on a triple antiretroviral therapy (ART) regimen. Of the HIV-positive women who had term deliveries, 47 (60\%) were on dual therapy, and $32(41 \%)$ were on ART.

Although $46 \%$ of the mothers had a CD4 count $\leq 350$ cells $/ \mathrm{mm}^{3}$, only $10 \%$ (three preterm deliveries and one term delivery) were on an ART regimen. The details are presented in Table 1.

Table 1: CD4 count versus treatment received during preterm deliveries

\begin{tabular}{|c|c|c|c|c|c|c|c|}
\hline \multirow[t]{3}{*}{ CD4 count } & \multicolumn{3}{|c|}{ Preterm deliveries } & \multicolumn{3}{|c|}{ Term } & \multirow[t]{2}{*}{ Total } \\
\hline & ART & DT & Total & ART & DT & Total & \\
\hline & $n, \%$ & $n, \%$ & $n, \%$ & $n, \%$ & $n, \%$ & $n, \%$ & $n, \%$ \\
\hline$\leq 350$ cells $/ \mathrm{mm}^{3}$ & $3(7.1)$ & $4(9.5)$ & $7(16.7)$ & $1(2.4)$ & $34(80.9)$ & $35(83.3)$ & $42(45.6)$ \\
\hline$>350$ cells $/ \mathrm{mm}^{3}$ & $1(2.0)$ & $5(10.0)$ & $6(12.0)$ & $31(62.0)$ & $13(26.0)$ & $44(88.0)$ & $50(54.4)$ \\
\hline Total & $4(30.8)$ & $9(69.2)$ & $13(100.0)$ & $32(40.5)$ & $47(59.5)$ & $79(100.0)$ & $92(100.0)$ \\
\hline
\end{tabular}

Note: ART: antiretroviral therapy, CD4: cluster of differentiation 4, DT: dual therapy

age, treatment received, co-morbidities and any other risk factors for preterm delivery. The condition of the preterm infants was determined with respect to acquired infections other than HIV during their hospital stay, perinatal mortality, hospital stay after delivery and Apgar scores.

The sample size was based on the assumption that $38 \%$ of the women presenting for delivery would be HIV positive (hospital statistics), of whom 7\% (hospital statistics) would be preterm, and that at least $20 \%$ of the HIV-positive group would be preterm. A group sample size of 96 HIV-positive women and 154 HIVnegative women was needed to achieve $80 \%$ power to detect a difference between the group proportions of $13 \%$. This was based on a two-tailed Fisher's exact test, with a significance level of $5 \%$.
Table 2: Risk factors associated with preterm deliveries

\begin{tabular}{|l|c|c|c|}
\hline Risk factors & OR & $95 \% \mathrm{CI}$ & $\boldsymbol{p}$ \\
\hline HIV status & 4.09 & $1.37-12.17$ & 0.01 \\
\hline Smoking & 12727924.12 & $0.00-0.00$ & 1.00 \\
\hline Alcohol & 20634398.70 & $0.00-0.00$ & 1.00 \\
\hline STI & 1.94 & $0.22-16.98$ & 0.55 \\
\hline UTI & 0.89 & $0.22-3.58$ & 0.87 \\
\hline Hypertension & 0.07 & $0.02-0.28$ & 0.00 \\
\hline Oligohydramnios & 0.00 & $0.00-0.00$ & 1.00 \\
\hline Multiple pregnancies & 0.00 & $0.00-0.00$ & 1.00 \\
\hline
\end{tabular}

Note: $\mathrm{Cl}$ : confidence interval, HIV: human immunodeficiency virus, OR: odds ratio, STI: sexually transmitted infection, UTI: urinary tract infection 
Table 3: Preterm neonatal outcomes

\begin{tabular}{|c|c|c|}
\hline Outcomes & $\begin{array}{l}\text { HIV exposed } \\
\qquad(n=14)\end{array}$ & $\begin{array}{l}\text { HIV unexposed } \\
\qquad(n=11)\end{array}$ \\
\hline Early neonatal death & 2 & 1 \\
\hline $\begin{array}{l}\text { Prolonged hospital stay of } \\
\geq 2 \text { weeks }\end{array}$ & 4 & 2 \\
\hline Hospital-acquired infection & 5 & 2 \\
\hline $\begin{array}{l}\text { An Apgar score of } \leq 7 \text { at } 5 \\
\text { minutes }\end{array}$ & 5 & 4 \\
\hline $\begin{array}{l}\text { Total number of adverse } \\
\text { outcomes }\end{array}$ & 16 & 9 \\
\hline
\end{tabular}

Note: HIV: human immunodeficiency virus

Multivariable logistic regression was performed to identify the risk factors associated with preterm delivery after adjustment for potential confounding. The results are depicted in Table 2.

Following multivariable adjustment, HIV-positive mothers were four times more likely to have a preterm delivery than their HIVnegative counterparts (OR 4.09, 95\% Cl: 1.37-12.17) $(p=0.010)$. This finding was highly significant.

\section{Outcomes of the preterm infants}

The adverse outcomes of the preterm births are detailed in Table 3 . Some neonates experienced more than one adverse clinical outcome. The number of neonates with an adverse outcome was too low for statistical significance to be inferred.

\section{Discussion}

\section{Maternal HIV and preterm delivery}

Of the 250 patients selected for this study, $37 \%$ of women were HIV positive. This is in line with a survey performed by the Department of Health in 2007, in which the prevalence of HIV infection in KwaZulu-Natal province was found to be $37 \%$, and which rose to $40 \%$ in $2010 .{ }^{16}$ The antenatal seroprevalence for HIV in KwaZulu-Natal was reported to be $38 \%$ in $2012 .{ }^{17}$ This indicates that there is a need to intensify efforts to reduce infection rates in women at every interaction that they have with the health service.

The prevalence of preterm delivery of $9 \%$ would be considered high by international standards, but not for the sub-Saharan African region. ${ }^{1}$ However, it is considered high for South Africa, which is regarded as an upper- to middle-income country; and cause for apprehension as preterm infants require more resources for care and support, which are limited in the public healthcare sector of South Africa. Preterm infants of HIV-infected mothers were found by Abrams et al. in 2004 to be at increased risk of morbidity and mortality and costly to the health system in Malawi. ${ }^{4}$ There was a fourfold risk of HIV-positive mothers having a preterm delivery than those who were HIV negative. This highlights the need to establish strategies to prevent premature deliveries and their associated complications by dealing effectively with HIV infection. These results are not dissimilar to the findings of a Nigerian study in which it was demonstrated that preterm deliveries occurred in $13 \%$ of HIV-positive mothers and $6 \%$ of HIV-negative mothers. ${ }^{6}$ The results confirm that maternal HIV infection is a significant risk factor for preterm delivery, supported by a study conducted by Lopez et al. in Barcelona, Spain, in which similar findings were reported. ${ }^{11}$ The relationship between HIV-infected women and preterm deliveries was also described in other studies carried out in Tanzania and the USA. ${ }^{10,12}$

Sexually transmitted infections (STIs) have been demonstrated to increase the risk of HIV infection, and it is assumed would increase the risk of a preterm delivery. ${ }^{8,18}$ Lee et al. demonstrated an association between concurrent syphilis with vertical perinatal HIV transmission, while Toskine et al. found that the prevalence of STIs was higher in HIV-infected women. ${ }^{8,18}$ However in this study, possibly because of the small sample size, STIs did not show a significant association with preterm deliveries.

Logistic regression analysis, used to adjust for potential confounders, showed that only HIV status (OR 4.09) and hypertension (OR 0.07) significantly influenced preterm deliveries in our study following multivariable adjustment. The association between HIV and preterm delivery has been established in other studies worldwide. ${ }^{4-6,11,12,19-21}$ However, the association between hypertension and preterm delivery demonstrated that preterm deliveries occurred significantly less often in women who had hypertension in pregnancy. This was reported previously in a review performed by Sibai. ${ }^{22}$ Generally, a district hospital manages patients with mild gestational hypertension, who are often admitted during the final weeks of pregnancy. Most cases of severe pre-eclampsia requiring urgent delivery are referred to regional and tertiary hospitals for further management. This may have accounted for the lower risk associated with preterm delivery and gestational hypertension found in this study.

\section{Neonatal outcomes}

Poor outcomes were reported in a large number of the preterm infants in our study. Sixteen adverse outcomes were found in 14 preterm infants in the HIV-exposed group, and nine poor outcomes in 11 preterm infants in the HIV-unexposed group. Ryder et al. noted that there was a significantly higher rate of complications due to prematurity in HIV-exposed infants than in the unexposed control group. ${ }^{9}$ This indicates that HIV-positive mothers are at higher risk of giving birth to preterm infants, whose health care is demanding and expensive, because of factors such as prolonged stay in the nursery and the treatment of associated infections. Problems relating to health cost are also mentioned in the study performed by Abrams et al., who showed that HIV infection was associated with poorer foetal outcomes, especially perinatal infections. ${ }^{23}$ Epalza et al. demonstrated that HIV-exposed infants were susceptible to group B streptococcal infection, but data on the cause of infection in preterm infants were not obtained in this study. ${ }^{7}$ A conclusion cannot be drawn on the increased risk of mortality in preterm HIV-exposed infants compared to the unexposed group owing to the small sample size, although it has been reported to be as high as 9.87 times greater when compared with its unexposed counterparts. ${ }^{13}$

Although HIV-exposed preterm neonates tended to stay in hospital for longer, the number of participants in the study was too small for a significant effect to be inferred. HIV infection has a significant impact on healthcare resources as preterm infants are nursed in a specialised nursery, with dedicated nursing care, for a long period. ${ }^{3} \mathrm{~A}$ difference in the number of low Apgar scores for preterm neonates born to HIV-positive, compared to HIVnegative, mothers could not be demonstrated in this study. However, a low Apgar score at five minutes was recorded in $36 \%$ of all of the preterm births, which usually indicates a poorer 
overall prognosis. ${ }^{3}$ The Apgar scores of the HIV-exposed infants might have been influenced by the associated clinical conditions of the pregnant mothers, as demonstrated in the study performed by Habib et al., who found that untreated HIVinfected women were at higher risk of experiencing an adverse pregnancy outcome, such as a low Apgar score and perinatal mortality. ${ }^{21}$

\section{Limitations of this study}

The study design was a retrospective cohort and did not have sufficient power to prove a causal link between pre-term HIV exposed neonates and neonatal outcomes. Data were missing in the clinical case files, and this may have introduced an element of information bias. The sample size of preterm deliveries in this study was small, so the results of this study cannot be generalised to a larger population.

\section{Recommendations}

Further studies should be performed to elaborate on the findings of this study.

A study with a large sample size and spanning a longer duration may also be able to demonstrate a closer association between preterm delivery and risk factors.

Medical staff need to improve their documentation on patients to ensure that comprehensive medical records are kept.

ART guidelines for pregnant women must be strictly followed.

The requirement in the new South African HIV guidelines that all pregnant women must be placed on an ART regimen may decrease the burden of preterm deliveries in HIV-positive women. ${ }^{24}$

A multipronged approach, in which prevention and treatment strategies are examined, is required to counteract the high HIV seroprevalence rate in pregnant women in KwaZulu-Natal.

\section{Conclusion}

This study aimed to explore the relationship between preterm deliveries and maternal HIV infection at an urban district hospital in KwaZulu-Natal. A significant association was made between preterm delivery and the HIV status of the pregnant women. A trend of more complications in HIV-exposed preterm neonates, than in HIV-unexposed preterm neonates, was also demonstrated in this study.

Acknowledgements - Prof Maurice Mars is thanked for his valuable input.

\section{References}

1. Beck S, Wojdyla D, Say L, et al. The worldwide incidence of preterm birth: a systematic review of maternal mortality and morbidity. Bull World Health Organ. 2010;88(1):31-8.

2. AVERT. South Africa HIV\&AIDS statistics 2010 [cited 2012 Mar 18]. Available from: http://www.avert.org/south-africa-hiv-aids-statistics. htm.

3. Blencowe H, Cousens S, Chou D, et al. Born Too Soon: the global epidemiology of 15 million preterm births. Reprod Health. 2013;10(Suppl 1):S2.

4. Abrams E, Milner D, Kwiek J, et al. Risk factors and mechanisms of preterm delivery in Malawi. Am J Reprod Immunol. 2004;52:174-83.

5. Ellis J, Williams $\mathrm{H}$, Graves $\mathrm{W}$, et al. Human immunodeficiency virus infection is a risk factor for adverse perinatal outcome. Am J Obstet Gynecol. 2002 May; 186(5):903-6.

6. Ezechi O, Gab-Okafor C, Oladele D, et al. Pregnancy, obstetric and neonatal outcomes in HIV positive Nigerian women. Afr J Reprod Health. 2013;17(3):160-8.

7. Epalza $C$, Goetghebuer T, Hainaut $M$, et al. High incidence of invasive group B streptococcal infections in HIV-exposed uninfected infants. Pediatrics. 2010 Sep;126(3):e631-8.

8. Toskine I, Verraes-Derancourt S, Letawe C, et al. Prevalence of asymptomatic sexual transmitted infection among high-risk patients attending a free anonymous HIV screening centre. Ann Dermatol Venereol. 2004;131:252-4.

9. Ryder RW, Nsuami M, Nsa W, et al. Mortality in HIV-1-seropositive women, their spouses and their newly born children during 36 months of follow-up in Kinshasa, Zaire. AIDS. 1994;8(5):667-72.

10. Ellis J, Williams $\mathrm{H}$, Graves W, et al. Human immunodeficiency virus infection is a risk factor for adverse perinatal outcome. Am J Obstet Gynecol. 2002;186(5):903-6.

11. Lopez M, Figueras F, Hernandez S, et al. Association of HIV infection with spontaneous and iatrogenic preterm delivery. AIDS. 2012;26(1):37-43.

12. Siza JE. Risk factors associated with low birth weight of neonates among pregnant women attending a referral hospital in Northern Tanzania. Tanzan J Health Res. 2008;10(1):3-11.

13. Fang LW, Xing ZL, Wang $L H$, et al. Influencing factors on the death of infants born to HIV infected mothers. Zhonghua Yu Fang Yi Xue Za Zhi [Chi J Prev Med]. 2009;43(11):991-5.

14. Taha TE, Dallabetta GA, Canner JK, et al. The effect of human immunodeficiency virus infection on birthweight, and infant and child mortality in urban Malawi. Int J Epidemiol. 1995;24(5):1022-9.

15. StataCorp. Stata Statistical Software: Release 13. College Station, TX: StataCorp LP; 2013.

16. AVERT. South Africa HIV\&AIDS statistics 2014 [cited 2014 Mar 5]. Available from: http://www.avert.org/south-africa-hiv-aids-statistics. htm.

17. Health indicators [Internet]. Health Systems Trust. 2014 [cited 2014 Oct 2]. Available from: http://indicators.hst.org.za/healthstats/13/ data.

18. Lee $M$, Hallmark R, Frenkel $L$, et al. Maternal syphilis and vertical perinatal transmission of human immunodeficiency virus type-1 infection. Int J Gynaecol Obst. 1998 Dec;63(3):247-52.

19. Abarzua F, Nunez F, Hubinont $C$, et al Human immunodeficiency virus (HIV) infection in pregnancy: antiretroviral treatment (ART) and mode of delivery. Rev Chil Infectol. 2005 [cited 2005 Dec 5];22(4):327-37.

20. Abdel-Latif M, Bajuk B, Oei J, et al. Does rural or urban residence make a difference to neonatal outcome in premature birth? A regional study in Australia Arch Dis Child Fetal Neonatal Ed. 2006;91(4):F251-6.

21. Habib NA, Daltveit AK, Bergsjø et al. Maternal HIV status and pregnancy outcomes in northeastern Tanzania: a registry-based study. Br J Obstet Gynaecol. 2008;115(5):616-24.

22. Sibai BM. Preeclampsia as a cause of preterm and late preterm (nearterm) births. Semin Perinatol. 2006;30(1):16-9.

23. Abrams $E$, Matheson $P$, Thomas $P$, et al. Neonatal predictors of infection status and early death among 332 infants at risk of HIV-1 infection monitored prospectively from birth. Pediatrics. 1995;96:451-9.

24. South African Department of Health. The South African antiretroviral treatment guidelines 2013. Pretoria; 2013 [cited 2013 Mar 14]. Available from: www.kznhealth.gov.za/medicine/2013_art_guidelines.pdf.

Received: 18-12-2014 Accepted: 10-06-2015 